\title{
Received Signal Strength Based Mobile Positioning via Constrained Weighted Least Squares
}

\author{
K. W. Cheung*, H. C. So*, W.-K. Ma\# and Y. T. Chan\# \\ *Department of Computer Eng. \& Information Technology, City University of Hong Kong, Tat Chee Avenue, Hong Kong \\ \#Department of Electronic Engineering, The Chinese University of Hong Kong, Shatin, N.T., Hong Kong
}

\begin{abstract}
Location estimation of mobile phones has received considerable interest in the field of wireless communications. In this paper, a simple and efficient positioning algorithm using received signal strength measurements obtained from at least three base stations are developed. Our proposed method is based on solving a nonconvex constrained weighted least squares problem. Simulation results show that the performance of the proposed method achieves the Cramér-Rao lower bound.
\end{abstract}

\section{Introduction}

Since the first ruling of the Federal Communication Commission for detection of emergency calls in the United States in 1996 [1], location estimation has become increasingly important for mobile services. Although the global positioning system (GPS) is widely utilized for positioning, it would be quite expensive to be adopted in the mobile phone network and its performance is poor when used in a densely populated area. Alternatively, using the base stations (BSs) in mobile network for localization is more desirable and less expensive. In addition to emergency management, the service will also be useful in monitoring children and the elderly, especially patients with Alzheimer's disease, intelligent transport systems, location billing and interactive map consultation [2]-[4].

Mobile phone positioning is often achieved by using the measurements of time of arrival (TOA), angle of arrival (AOA), time difference of arrival (TDOA) or received signal strength (RSS). Since most of the European countries are now using the Global System for Mobile Communications (GSM), which provides only the RSS measurements, we will focus on positioning using the RSS measurements in this paper. However, our proposed methodology can be easily extended to TOA-based and TDOA-based location systems.

In the RSS method, the propagation path losses from the mobile station (MS) to the BSs are measured, which are then converted to distances between them. For twodimensional (2D) positioning, each RSS measurement will provide a circle centered at the corresponding BS, on which the MS must lie. In the absence of measurement error, the MS position is given by the intersection of circles from at least three BSs in order to resolve ambiguities arising from multiple crossings of the lines of position. In practical situations when the RSS measurements are in errors, nonlinear least squares is an appropriate but computationally intensive approach for MS positioning. The main idea of the proposed algorithm is to transform the nonlinear equations relating the RSS measurements to the MS location into a set of linear equations by introducing an extra range variable, which is adopted from [5]-[7] in TDOA-based systems. The linear equations are then solved by weighted least squares (WLS) subject to the relation between the range parameter and the MS position coordinates.

The rest of this paper is organized as follows. The model for RSS measurements is described in Section II. In Section III, the proposed algorithm that weighs the least squares function and exploits the relation between the range parameter and the position coordinates is devised. In Section IV, the CRLB is derived. Simulation results are presented in Section V. Finally, conclusions are drawn in Section VI.

\section{RSS Measurement Model}

It is assumed that a reliable non-line-of-sight (NLOS) detection algorithm [8] has first been employed to eliminate the measurements with large errors. As a result, all measurements we utilize for the MS location come from line-of-sight (LOS) propagation. Let the true location of MS be $\left[x_{s}, y_{s}\right]^{T}$ and the coordinates of the $i^{t h}$ BS be $\left[x_{i}, y_{i}\right]^{T}, i=1,2, \ldots, N$, where $N$ is the total number of receiving LOS BSs. The distance between the MS and the $i^{\text {th }} \mathrm{BS}$, denoted by $d_{i}$, is given by

$$
d_{i}=\sqrt{\left(x_{s}-x_{i}\right)^{2}+\left(y_{s}-y_{i}\right)^{2}}, \quad i=1,2, \ldots, N
$$

The noise-free RSS or received power at the $i^{\text {th }}$ BS, denoted by $P_{i}^{r}$, is expressed as [9]

$$
P_{i}^{r}=K_{i} \frac{P_{i}^{t}}{d_{i}^{a}}, \quad i=1,2, \ldots, N
$$

where $P_{i}^{t}$ is the transmitted power, $K_{i}$ is the rest of all other factors which affects the received power including the antenna height and antenna gain, and $a$ is the propagation constant. The range-related measurement which uses the known $P_{i}^{t}, K_{i}$ and the measured RSS, denoted by $r_{i}$, is modelled as

$r_{i}=K_{i} \frac{P_{i}^{t}}{P_{i}^{r}}+n_{i}$ 


$$
\begin{aligned}
& =d_{i}^{a}+n_{i} \\
& =\left[\left(x_{s}-x_{i}\right)^{2}+\left(y_{s}-y_{i}\right)^{2}\right]^{\frac{a}{2}}+n_{i}, \quad i=1,2, \ldots, N
\end{aligned}
$$

where $n_{i}$ is the noise in $r_{i}$ at the $i^{t h}$ BS. The propagation parameter $a$ can be obtained by finding the path loss slope by measurement. In free space, $a$ is equal to 2 , but in some urban and suburban areas, $a$ can be from 3 to 6 . The unit of the measurement $r_{i}$ is $m^{a}$.

For ease of analysis, we assume that measurement errors $\left\{n_{i}\right\}$ are zero mean white Gaussian process with corresponding variances $\left\{\sigma_{i}^{2}\right\}$. The probability density function of $\mathbf{r}=\left[r_{1}, r_{2}, \ldots, r_{N}\right]^{T}$ conditioned on the MS position $\mathbf{u}=\left[x_{s}, y_{s}\right]^{T}$ is given by

$$
\begin{aligned}
& p(\mathbf{r} \mid \mathbf{u})=\frac{1}{\sqrt{(2 \pi)^{N} \prod_{i=1}^{N} \sigma_{i}^{2}}} \cdot \\
& \quad \exp \left\{\sum_{i=1}^{N} \frac{1}{2 \sigma_{i}^{2}}\left[r_{i}-\left[\left(x_{s}-x_{i}\right)^{2}+\left(y_{s}-y_{i}\right)^{2}\right]^{\frac{a}{2}}\right]^{2}\right\}
\end{aligned}
$$

\section{Constrained WLS Algorithm}

In this section, we introduce the proposed algorithm for RSS-based mobile positioning. Without measurement errors, (3) becomes

$$
r_{i}=\left[\left(x_{s}-x_{i}\right)^{2}+\left(y_{s}-y_{i}\right)^{2}\right]^{\frac{a}{2}}, \quad i=1,2, \ldots, N
$$

Taking power $\frac{2}{a}$ on both sides of (5) yields

$$
\begin{aligned}
r_{i}^{\frac{2}{a}}=R_{s}^{2}-2 x_{s} x_{i}-2 y_{s} y_{i}+ & \left(x_{i}^{2}+y_{i}^{2}\right) \\
\Rightarrow x_{i} x_{s}+y_{i} y_{s}-0.5 R_{s}^{2}= & \frac{1}{2}\left(x_{i}^{2}+y_{i}^{2}-r_{i}^{\frac{2}{a}}\right), \\
& i=1,2, \ldots, N
\end{aligned}
$$

where $R_{s}=\sqrt{x_{s}^{2}+y_{s}^{2}}$ is the intermediate variable in order to linearize (5) in terms of $x_{s}, y_{s}$ and $R_{s}^{2}$. Eq. (6) can be expressed in a matrix form

$$
\mathbf{A} \theta=\mathbf{b}
$$

where

$$
\mathbf{A}=\left[\begin{array}{ccc}
x_{1} & y_{1} & -0.5 \\
\vdots & \vdots & \vdots \\
x_{N} & y_{N} & -0.5
\end{array}\right], \boldsymbol{\theta}=\left[\begin{array}{c}
x_{s} \\
y_{s} \\
R_{s}^{2}
\end{array}\right]
$$

and

$$
\mathbf{b}=\frac{1}{2}\left[\begin{array}{c}
x_{1}^{2}+y_{1}^{2}-r_{1}^{\frac{2}{a}} \\
\vdots \\
x_{N}^{2}+y_{N}^{2}-r_{N}^{\frac{2}{a}}
\end{array}\right]
$$

In the presence of measurement errors, $\theta$ can be estimated using the standard least squares as

$$
\begin{aligned}
\hat{\boldsymbol{\theta}} & =\underset{\boldsymbol{\theta}}{\arg \min _{\boldsymbol{\theta}}}(\mathbf{A} \boldsymbol{\theta}-\mathbf{b})^{\mathrm{T}}(\mathbf{A} \boldsymbol{\theta}-\mathbf{b}) \\
& =\left(\mathbf{A}^{\mathrm{T}} \mathbf{A}\right)^{-\mathbf{1}} \mathbf{A}^{\mathrm{T}} \mathbf{b}
\end{aligned}
$$

In order to achieve better performance, we should add a weighting matrix to (8) and restrict $\boldsymbol{\theta}$ to the basic relationship

$$
R_{s}^{2}=x_{s}^{2}+y_{s}^{2}
$$

The resulting location estimator is a constrained optimization problem as follows.

$$
\hat{\boldsymbol{\theta}}=\arg \min _{\boldsymbol{\theta}}(\mathbf{A} \boldsymbol{\theta}-\mathbf{b})^{\mathrm{T}} \mathbf{W}(\mathbf{A} \boldsymbol{\theta}-\mathbf{b})
$$

subject to

$$
\mathrm{q}^{\mathrm{T}} \boldsymbol{\theta}+\boldsymbol{\theta}^{\mathrm{T}} \mathbf{P} \boldsymbol{\theta}=0
$$

where

$$
\mathbf{P}=\left[\begin{array}{lll}
1 & 0 & 0 \\
0 & 1 & 0 \\
0 & 0 & 0
\end{array}\right] \text { and } \mathbf{q}=\left[\begin{array}{c}
0 \\
0 \\
-1
\end{array}\right]
$$

We can easily see that (11) is a matrix representation of (9).

In order to find the weighting matrix $\mathbf{W}$, the disturbance in $\mathbf{b}$ is studied. Assuming sufficiently small measurement error, the value $r_{i}^{\frac{2}{a}}$ can be approximated using Taylor series as

$$
\begin{aligned}
r_{i}^{\frac{2}{a}} & =\left(d_{i}^{a}+n_{i}\right)^{\frac{2}{a}} \\
& \approx d_{i}^{2}+\frac{2}{a}\left(d_{i}\right)^{2-a} n_{i} \quad i=1,2, \ldots, N
\end{aligned}
$$

As a result, the disturbance between the true and estimate of the squared distances is

$$
\varepsilon_{i}=r_{i}^{\frac{2}{a}}-d_{i}^{2} \approx \frac{2}{a}\left(d_{i}\right)^{2-a} n_{i} \quad i=1,2, \ldots, N
$$

In vector form, $\left\{\varepsilon_{i}\right\}$ is expressed as

$$
\varepsilon=\left[\frac{2}{a}\left(d_{1}\right)^{2-a} n_{1}, \frac{2}{a}\left(d_{2}\right)^{2-a} n_{2}, \ldots, \frac{2}{a}\left(d_{N}\right)^{2-a} n_{N}\right]^{T}
$$

The covariance matrix of the disturbance is thus of the form

$$
\boldsymbol{\Psi}=E\left\{\varepsilon \varepsilon^{T}\right\}=\mathbf{B Q} \mathbf{B}
$$

where $\mathbf{B}=\operatorname{diag}\left\{\frac{2}{a} d_{1}^{2-a}, \frac{2}{a} d_{2}^{2-a}, \ldots, \frac{2}{a} d_{N}^{2-a}\right\}$ and $\mathbf{Q}=$ $\operatorname{diag}\left\{\sigma_{1}^{2}, \sigma_{2}^{2}, \ldots, \sigma_{N}^{2}\right\}$. Under the above assumptions, the optimum weighting matrix for (10) is $\mathbf{W}=$ 
$\boldsymbol{\Psi}^{-1}$, which depends on the unknown $\left\{d_{i}\right\}$. For this reason, we approximate $\boldsymbol{\Psi} \approx \hat{\mathbf{B}} \mathbf{Q} \hat{\mathbf{B}}$, where $\hat{\mathbf{B}}=$ $\operatorname{diag}\left\{\frac{2}{a} r_{1}^{2 / a-1}, \frac{2}{a} r_{2}^{2 / a-1}, \ldots, \frac{2}{a} r_{N}^{2 / a-1}\right\}$.

We now consider solving (10) subject to (11), which is equivalent to minimizing the Lagrangian

$\mathcal{L}(\boldsymbol{\theta}, \lambda)=(\mathbf{A} \boldsymbol{\theta}-\mathbf{b})^{\mathrm{T}} \boldsymbol{\Psi}^{-\mathbf{1}}(\mathbf{A} \boldsymbol{\theta}-\mathbf{b})+\lambda\left(\mathbf{q}^{\mathrm{T}} \boldsymbol{\theta}+\boldsymbol{\theta}^{\mathrm{T}} \mathbf{P} \boldsymbol{\theta}\right)$

where $\lambda$ is the Lagrange multiplier. The minimum of (16) is obtained by differentiating $\mathcal{L}(\boldsymbol{\theta}, \lambda)$ with respect to $(\theta, \lambda)$ and then equating the resultant expressions to zero:

$$
\frac{\partial \mathcal{L}(\boldsymbol{\theta}, \lambda)}{\partial \boldsymbol{\theta}}=2\left(\mathbf{A}^{\mathbf{T}} \boldsymbol{\Psi}^{-\mathbf{1}} \mathbf{A}+\lambda \mathbf{P}\right) \boldsymbol{\theta}-2 \mathbf{A}^{\mathbf{T}} \boldsymbol{\Psi}^{-\mathbf{1}} \mathbf{b}+\lambda \mathbf{q}=0
$$

$$
\frac{\partial \mathcal{L}(\boldsymbol{\theta}, \lambda)}{\partial \lambda}=\mathbf{q}^{\mathrm{T}} \boldsymbol{\theta}+\boldsymbol{\theta}^{\mathrm{T}} \mathbf{P} \boldsymbol{\theta}=0
$$

Solving for $\theta$ by (17) yields the constrained WLS estimate

$$
\hat{\boldsymbol{\theta}}(\lambda)=\left(\mathbf{A}^{\mathrm{T}} \boldsymbol{\Psi}^{-\mathbf{1}} \mathbf{A}+\lambda \mathbf{P}\right)^{-\mathbf{1}}\left(\mathbf{A}^{\mathrm{T}} \boldsymbol{\Psi}^{-\mathbf{1}} \mathbf{b}-\frac{\lambda}{\mathbf{2}} \mathbf{q}\right)
$$

where $\lambda$ is not determined yet. In order to find $\lambda$, we substitute (19) into (18)

$$
\begin{array}{r}
\mathbf{q}^{\mathrm{T}}\left(\mathbf{A}^{\mathrm{T}} \boldsymbol{\Psi}^{-1} \mathbf{A}+\lambda \mathbf{P}\right)^{-\mathbf{1}}\left(\mathbf{A}^{\mathrm{T}} \boldsymbol{\Psi}^{-1} \mathbf{b}-\frac{\lambda}{\mathbf{2}} \mathbf{q}\right) \\
+\left(\mathbf{b}^{\mathrm{T}} \boldsymbol{\Psi}^{-\mathbf{1}} \mathbf{A}-\frac{\lambda}{\mathbf{2}} \mathbf{q}^{\mathrm{T}}\right)\left(\mathbf{A}^{\mathrm{T}} \boldsymbol{\Psi}^{-\mathbf{1}} \mathbf{A}+\lambda \mathbf{P}\right)^{-\mathbf{1}} \mathbf{P} \\
\left(\mathbf{A}^{\mathrm{T}} \boldsymbol{\Psi}^{-\mathbf{1}} \mathbf{A}+\lambda \mathbf{P}\right)^{-\mathbf{1}}\left(\mathbf{A}^{\mathrm{T}} \boldsymbol{\Psi}^{-\mathbf{1}} \mathbf{b}-\frac{\lambda}{\mathbf{2}} \mathbf{q}\right)=0
\end{array}
$$

The matrix $\left(\mathbf{A}^{\mathbf{T}} \boldsymbol{\Psi}^{-\mathbf{1}} \mathbf{A}\right)^{-\mathbf{1}} \mathbf{P}$ can be diagonalized as

$$
\left(\mathbf{A}^{\mathrm{T}} \boldsymbol{\Psi}^{-1} \mathbf{A}\right)^{-1} \mathbf{P}=\mathbf{U} \Lambda \mathbf{U}^{-1}
$$

where $\boldsymbol{\Lambda}=\operatorname{diag}\left(\gamma_{1}, \gamma_{2}, \gamma_{3}\right)$ and $\gamma_{i}, i=1,2,3$ are the eigenvalues of the matrix $\left(\mathbf{A}^{\mathbf{T}} \boldsymbol{\Psi}^{-\mathbf{1}} \mathbf{A}\right)^{-\mathbf{1}} \mathbf{P}$. Substituting (21) into $\left(\mathbf{A}^{\mathbf{T}} \boldsymbol{\Psi}^{-\mathbf{1}} \mathbf{A}\right)^{-\mathbf{1}} \mathbf{P}$ gives

$$
\left(\mathbf{A}^{\mathrm{T}} \boldsymbol{\Psi}^{-\mathbf{1}} \mathbf{A}+\lambda \mathbf{P}\right)^{-\mathbf{1}}=\mathbf{U}(\mathbf{I}+\lambda \mathbf{\Lambda})^{-1} \mathbf{U}^{-1}\left(\mathbf{A}^{\mathrm{T}} \boldsymbol{\Psi}^{-1} \mathbf{A}\right)^{-\mathbf{1}}
$$

Putting (22) into (20), we get

$$
\begin{aligned}
& \mathbf{c}^{\mathrm{T}}(\mathbf{I}+\lambda \boldsymbol{\Lambda})^{-\mathbf{1}} \mathbf{f}-\frac{\lambda}{\mathbf{2}} \mathbf{c}^{\mathrm{T}}(\mathbf{I}+\lambda \boldsymbol{\Lambda})^{-1} \mathrm{~g}+\mathbf{e}^{\mathrm{T}}(\mathbf{I}+\lambda \boldsymbol{\Lambda})^{-1} \\
& \boldsymbol{\Lambda}(\mathbf{I}+\lambda \boldsymbol{\Lambda})^{-\mathbf{1}} \mathbf{f}-\frac{\lambda}{\mathbf{2}} \mathrm{e}^{\mathrm{T}}(\mathrm{I}+\lambda \boldsymbol{\Lambda})^{-\mathbf{1}} \boldsymbol{\Lambda}(\mathrm{I}+\lambda \boldsymbol{\Lambda})^{-\mathbf{1}} \mathrm{g} \\
& -\frac{\lambda}{2} c^{\mathrm{T}}(\mathbf{I}+\lambda \boldsymbol{\Lambda})^{-1} \boldsymbol{\Lambda}(\mathbf{I}+\lambda \boldsymbol{\Lambda})^{-\mathbf{1}} \mathbf{f} \\
& +\frac{\lambda^{2}}{4} \mathrm{c}^{\mathrm{T}}(\mathrm{I}+\lambda \boldsymbol{\Lambda})^{-1} \Lambda(\mathrm{I}+\lambda \boldsymbol{\Lambda})^{-1} \mathrm{~g}=0
\end{aligned}
$$

where

$\mathbf{c}^{\mathrm{T}}=\mathbf{q}^{\mathrm{T}} \mathbf{U}=\left[c_{1}, c_{2}, c_{3}\right], \mathrm{g}=\mathbf{U}^{-\mathbf{1}}\left(\mathbf{A}^{\mathrm{T}} \boldsymbol{\Psi}^{-\mathbf{1}} \mathbf{A}\right)^{-\mathbf{1}}$, $q=\left[g_{1}, g_{2}, g_{3}\right]^{T}, \mathbf{e}^{\mathbf{T}}=\mathbf{b}^{\mathbf{T}} \boldsymbol{\Psi}^{-\mathbf{1}} \mathbf{A} \mathbf{U}=\left[e_{1}, e_{2}, e_{3}\right]$ and $\mathbf{f}=\mathbf{U}^{-1}\left(\mathbf{A}^{\mathbf{T}} \boldsymbol{\Psi}^{-1} \mathbf{A}\right)^{-\mathbf{1}} \mathbf{A} \boldsymbol{\Psi}^{-1} \mathbf{b}=\left[f_{1}, f_{2}, f_{3}\right]^{T}$.

Since the matrix $\left(\mathbf{A}^{\mathbf{T}} \mathbf{\Psi}^{-\mathbf{1}} \mathbf{A}\right)^{-\mathbf{1}} \mathbf{P}$ is of rank 2 , one of its eigenvalues, say, $\gamma_{3}$, must be zero. After expansion of equation (23) and putting $\gamma_{3}=0,(23)$ is simplified to

$$
\begin{aligned}
& c_{3} f_{3}-\frac{\lambda}{2} c_{3} g_{3}+\sum_{i=1}^{2} \frac{c_{i} f_{i}}{1+\lambda \gamma_{i}}-\frac{\lambda}{2} \sum_{i=1}^{2} \frac{c_{i} g_{i}}{1+\lambda \gamma_{i}} \\
& +\sum_{i=1}^{2} \frac{e_{i} f_{i} \gamma_{i}}{\left(1+\lambda \gamma_{i}\right)^{2}}-\frac{\lambda}{2} \sum_{i=1}^{2} \frac{e_{i} g_{i} \gamma_{i}}{\left(1+\lambda \gamma_{i}\right)^{2}} \\
& -\frac{\lambda}{2} \sum_{i=1}^{2} \frac{c_{i} f_{i} \gamma_{i}}{\left(1+\lambda \gamma_{i}\right)^{2}}+\frac{\lambda^{2}}{4} \sum_{i=1}^{2} \frac{c_{i} g_{i} \gamma_{i}}{\left(1+\lambda \gamma_{i}\right)^{2}}=0(24)
\end{aligned}
$$

which is an equation of 5 roots. The desired $\lambda$ is found by the following procedures.

i. Find the 5 roots by using a standard root finding algorithm. Then take only the real roots into consideration as the Lagrange multiplier is always real for real optimization problems.

ii. Put the real $\lambda$ 's back to (19) and obtain subestimates of $\boldsymbol{\theta}$.

iii. Substitute the sub-estimates into $(\mathbf{A} \boldsymbol{\theta}-\mathbf{b})^{\mathrm{T}} \mathbf{W}(\mathbf{A} \boldsymbol{\theta}-\mathbf{b})$ and the sub-estimate that makes the expression minimum is the solution $\hat{\boldsymbol{\theta}}$.

\section{Cramér-Rao Lower Bound}

The Cramér-Rao lower bound (CRLB) gives a lower bound on variance attainable by any unbiased estimators and thus it can be served as a benchmark to contrast with the mean square error of the RSS positioning algorithms. The CRLB for the $k^{t h}$ parameter estimate of $\mathbf{u}$, denoted by $\operatorname{CRLB}\left(\hat{u}_{k}\right)$ can be computed from

$$
\operatorname{CRLB}\left(\hat{u}_{k}\right)=\left[\mathbf{I}^{-\mathbf{1}}(\mathbf{u})\right]_{k k}
$$

where

$$
[\mathbf{I}(\mathbf{u})]_{i j}=-E\left[\frac{\partial^{2} \ln p(\mathbf{r} \mid \mathbf{u})}{\partial u_{i} \partial u_{j}}\right]
$$

is the corresponding Fisher information matrix (FIM), $\hat{\mathbf{u}}=\left[\hat{u}_{1}, \hat{u}_{2}\right]^{T}=\left[\hat{x}_{s}, \hat{y}_{s}\right]^{T}$ is the location estimate of the MS and $E[$.$] is the expectation operator. From (26), the$ FIM for the RSS location technique can be calculated as

$$
\mathbf{I}(\mathbf{u})=
$$

$\left[\begin{array}{c}\sum_{i=1}^{N} \frac{a^{2}\left(x_{s}-x_{i}\right)^{2}}{\sigma_{i}^{2}\left[\left(x_{s}-x_{i}\right)^{2}+\left(y_{s}-y_{i}\right)^{2}\right]^{2-a}} \sum_{i=1}^{N} \frac{a^{2}\left(x_{s}-x_{i}\right)\left(y_{s}-y_{i}\right)}{\sigma_{i}^{2}\left[\left(x_{s}-x_{i}\right)^{2}+\left(y_{s}-y_{i}\right)^{2}\right]^{2-a}} \\ \sum_{i=1}^{N} \frac{a^{2}\left(x_{s}-x_{i}\right)\left(y_{s}-y_{i}\right)}{\sigma_{i}^{2}\left[\left(x_{s}-x_{i}\right)^{2}+\left(y_{s}-y_{i}\right)^{2}\right]^{2-a}} \sum_{i=1}^{N} \frac{a^{2}\left(y_{s}-y_{i}\right)^{2}}{\sigma_{i}^{2}\left[\left(x_{s}-x_{i}\right)^{2}+\left(y_{s}-y_{i}\right)^{2}\right]^{2-a}}\end{array}\right]$

Then the corresponding CRLB can be calculated by (25) and (27). It is noteworthy that the CRLB should remain unchanged [10] when the NLOS measurements, if any, are also included in the computation. 


\section{Simulation Results}

Computer simulations were performed to evaluate the performance of the proposed RSS-based location algorithms for the cases of 3 and 7 BSs. For $7 \mathrm{BSs}$ case, the location of BSs were situated at $[0,0] \mathrm{m},[3000 \sqrt{3}, 3000] \mathrm{m},[0,6000] \mathrm{m}$, $[-3000 \sqrt{3}, 3000] \mathrm{m},[-3000 \sqrt{3},-3000] \mathrm{m},[0,-6000] \mathrm{m}$ and $[3000 \sqrt{3},-3000] \mathrm{m}$. Only the first three BSs were involved for the case of $3 \mathrm{BSs}$ and $a=2$ was chosen which corresponded to a free space propagation. The location of MS was at $[-100,200] \mathrm{m}$. All results were based on 1000 independent runs.

Figure 1 shows the mean square range errors (MSREs), defined as $E\left[\left(x_{s}-\hat{x_{s}}\right)^{2}+\left(y_{s}-\hat{y}_{s}\right)^{2}\right]$, of the standard LS method, the constrained WLS method as well as the CRLB versus the average SNR, given by $\frac{1}{N} \sum_{i=1}^{N} \frac{d_{i}^{2}}{\sigma_{i}^{2}}$, for the 3 -BS case. In this simulation, the SNRs were identical for all RSS measurements. It can be seen that the performance of the standard LS was inferior to the constrained WLS by about $2 \mathrm{~dB}$ which was very close to optimum. The above test was repeated for the case of 7 BSs and the results are shown in Figure 2. We observe that the constrained WLS slightly outperformed the standard LS and still achieved the CRLB. It is also noted that MSREs for 7 BSs were always lower than those for $3 \mathrm{BSs}$. Therefore, the performance of the proposed method was better when more BSs were utilized for mobile positioning.

\section{Conclusions}

A simple and efficient mobile positioning algorithm using received signal strength (RSS) measurements is proposed. The idea is to transform the nonlinear equations relating the RSS to the mobile position to a set of linear equations, which are then solved by constrained weighted least squares. It is shown by simulations that the proposed method approaches the optimum location accuracy.

\section{Acknowledgement}

The work described in this paper was partially supported by a grant from the Research Grants Council of the Hong Kong Special Administrative Region, China [Project No. CityU 1119/01E].

\section{References}

[1] http://www.fcc.gov/e911

[2] H.Koshima and J.Hosen, "Personal locator services emerge," IEEE Spectrum, pp.41-48, Feb. 2000

[3] D.Porcino, "Performance of a OTDOA-IPDL positioning receiver for $3 \mathrm{G}-\mathrm{FDD}$ mode," Proc. Int. Conf. $3 G$ Mobile Communication Technologies, pp.221-225, 2001

[4] J.J.Caffery, Jr., Wireless Location in CDMA Cellular Radio Systems, Kluwer Academic Publishers, 2000
[5] J.O.Smith and J.S.Abel, "Closed-form least-squares source location estimation from range-difference measurements," IEEE Trans. Acoust., Speech, Signal Processing, vol.35, pp. 1661-1669, Dec. 1987

[6] Y.T.Chan and K.C.Ho, "A simple and efficient estimator for hyberbolic location," IEEE Trans. Signal Processing, vol.42, pp. 1905-1915, Aug. 1994

[7] Y.Huang, J.Benesty, G.W.Elko and R.M.Mersereau, "Real-time passive source localization: a practical linear-correction least-squares approach," IEEE Trans. Speech, Audio Processing, vol.9, pp.943-956, Nov 2001 [8] S.Al-Jazzar, J.Caffery, Jr. and H.R.You, " A scattering model based approach to NLOS mitigation in TOA location systems," Proc. IEEE 55th Vehicular Technology Conference, vol.2, pp.861-865, Spring 2002 [9] H.L.Song, "Automatic vehicle location in cellular communications systems", IEEE Trans. Vehicular Technology, vol.43, pp. 902-908, Nov. 1994

[10] Y.Qi and H.Kobayashi, "Cramér-Rao lower bound for geolcation in non-line-of-sight environment," Proc. ICASSP 2002, vol.3, pp.2473-2476, 2002

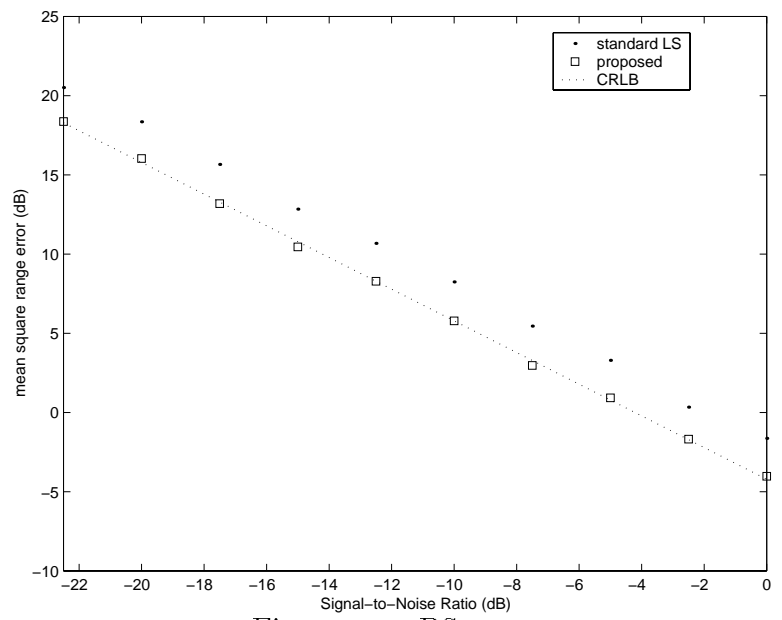

Figure 1: 3-BS case

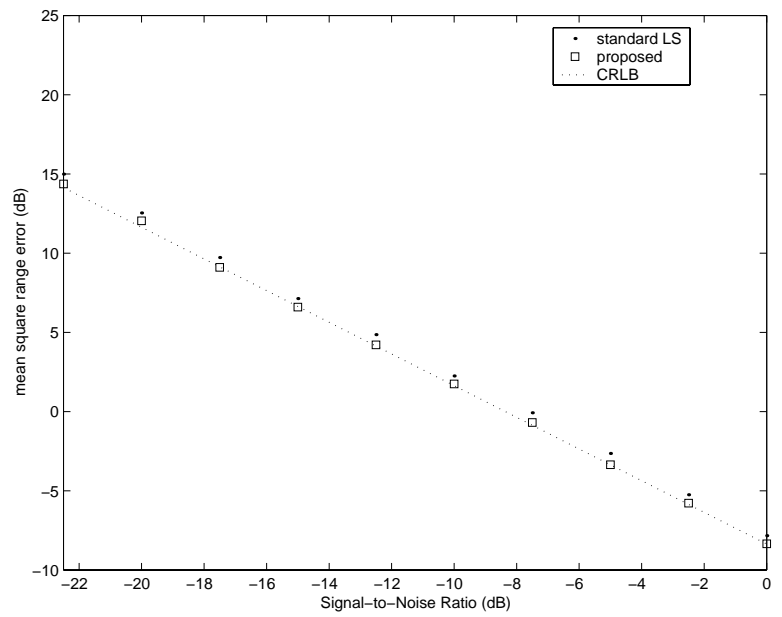

Figure 2: 7-BS case 